\title{
ANÁLISE COMPARATIVA DA PRECIPITAÇÃO NO ESTADO DA PARAÍBA UTILIZANDO MODELOS DE REGRESSÃO POLINOMIAL
}

\author{
OSEAS MACHADO GOMES ${ }^{1}$, CARLOS ANTONIO COSTA DOS SANTOS ${ }^{2}$, FRANCISCO DE \\ ASSIS SALVIANO DE SOUZA ${ }^{2}$, WILLIAN DE PAIVA ${ }^{1}$, RICARDO ALVES DE OLINDA ${ }^{1}$ \\ ${ }^{1}$ Universidade Estadual da Paraíba (UEPB), Departamento de Estatística, Campina Grande, PB, Brasil \\ ${ }^{2}$ Universidade Federal de Campina Grande (UFCG), Unidade Acadêmica de Ciências Atmosféricas, \\ Campina Grande, PB, Brasil
}

\begin{abstract}
oseasmachado@gmail.com, carlostorm@gmail.com, fassis@dca.ufcg.edu.br,wili123@ig.com.br, ricardo.estat@yahoo.com.br
\end{abstract}

Recebido Março de 2012 - Aceito Julho de 2014

\begin{abstract}
RESUMO
O Estado da Paraíba apresenta alta variabilidade espacial e temporal na distribuição das chuvas. Além disso, existem muitos locais onde não se tem informações sobre os valores de precipitação. Logo, o uso da regressão polinomial (superfície de tendência) na tentativa de obter estimativas de precipitação pode ser uma alternativa para um melhor entendimento da variabilidade espaço-temporal desse parâmetro meteorológico. Assim, o objetivo principal desse estudo foi utilizar o método da regressão polinomial como ferramenta para estimar médias mensais de precipitação para locais conhecidos (estações meteorológicas ou postos pluviométricos) e compará-las com valores medidos, com o intuito de verificar sua precisão dentro da região de estudos. Foram utilizados dados médios mensais de precipitação pluviométrica provenientes de 102 estações meteorológicas distribuídas espacialmente no estado da Paraíba, no período de 1962 a 2001. Os dados apresentaram alta variabilidade espacial em todo o Estado. As regressões polinomiais que melhor se ajustaram aos dados observados foram as de $3^{\mathrm{a}}$ e $4^{\mathrm{a}}$ ordens, e a comparação entre os valores de precipitação medidos e estimados mostraram que os meses de junho a novembro apresentaram as menores diferenças de precipitação na maior parte do território paraibano. Sendo assim, o método adotado se apresenta como uma ferramenta promissora na estimativa da precipitação mensal no Estado da Paraíba.
\end{abstract}

Palavras-chave: superfície de tendência; variabilidade espacial; análise de variância.

\begin{abstract}
COMPARATIVE ANALYSIS OF RAINFALL IN THE STATE OF PARAÍBA USING POLYNOMIAL REGRESSION MODELS

The State of Paraíba shows high spatial and temporal variability in rainfall distribution. In addition, there are many locations with no information about the precipitation values. Thus, the use of polynomial regression (trend surface) in an attempt to obtain estimates of precipitation can be an alternative to a better understanding of the spatiotemporal variability of this meteorological parameter. Thus, the main objective of this study was to use the method of polynomial regression as a tool to estimate monthly averages of rainfall at known locations (weather and rain gauges stations) and compare them with measured values, in order to verify its accuracy within the region of interest. Monthly precipitation data series from 102 weather stations distributed throughout the State of Paraíba, in the period 19622001 have been used. The data showed high spatial variability across the state. The $3^{\text {rd }}$ and $4^{\text {th }}$ order polynomial regressions have best fit the observed data and the comparison between measured and estimated precipitation values showed that the months from June to November showed the smallest differences over almost the Paraíba territory. Thus, the method adopted is presented as a promising tool to estimate monthly rainfall in the State of Paraíba.
\end{abstract}

Keywords: trend surface; spatial variability; analysis of variance. 


\section{INTRODUÇÃO}

A Paraíba está situada entre os paralelos $6^{\circ}$ e $8^{\circ}$ graus de latitude sul, e entre os meridianos de $34^{\circ}$ e $39^{\circ}$ graus de longitude oeste, totalmente incluída na Região Tropical e faz divisa com os estados de Pernambuco, Rio Grande do Norte e Ceará, limitando-se à leste com o Oceano Atlântico. Tem uma área de aproximadamente $56.340,9 \mathrm{~km}^{2}$ com quatro mesorregiões e 223 municípios (Rodriguez, 2002). O índice de precipitação no estado da Paraíba depende de vários sistemas meteorológicos tais como: Zona de Convergência Intertropical (ZCIT), Vórtices Ciclônicos de Altos Níveis (VCAN), Sistemas Ondulatórios de Leste, Linhas de Instabilidade, Sistemas Frontais, brisas terrestre e marítima, bandas de nebulosidade, assim como, efeitos orográficos locais (Macedo et al., 2010), que atuam em diferentes meses do ano com características interanuais diferentes, fazem com que haja grande variabilidade das chuvas na direção Norte - Sul e Leste - Oeste durante todo ano (Pedroza, 2009). Devido a essa variabilidade é de interesse, por parte dos pesquisadores, ajustar modelos que possam estimar valores de precipitação para todo Estado, devido à existência de poucas estações meteorológicas distribuídas em seu território, conforme apresentado na Figura 1.

Além da alta variabilidade espacial e temporal na distribuição das chuvas no Estado da Paraíba, existem muitos locais dos quais não se tem informações sobre o índice de precipitação. Para suprir essa necessidade, pode-se utilizar a regressão polinomial (superfície de tendência) na tentativa de obter estimativas de precipitação e compará-las com valores observados, a fim de verificar a adequabilidade do modelo aos dados. A regressão polinomial é um método de interpolação, que permite inferir ou estimar características de interesse sobre os dados, tanto em locais conhecidos (estações meteorológicas ou postos pluviométricos), como desconhecidos dentro da região de estudos.

Segundo Landim e Corsi (2001) a técnica é ajustada pelo método dos mínimos quadrados, em que $Z_{i}$ é a variável dependente e $(X, Y)$ são as variáveis independentes representadas pelas coordenadas Norte - Sul $(Y)$ e Leste Oeste $(X)$, minimizando-se, assim, diferenças de quadrados entre a superfície e os pontos medidos (EPA, 2004). Tendo em vista que as estações meteorológicas encontram-se numa grid irregular (Figura 1), a aplicação da técnica baseou-se nos polinômios não ortogonais adicionando-se termos à equação polinomial, observando-se, por meio de critérios estatísticos, a superfície que melhor se ajusta aos dados. Além disso, devido o método gerar o melhor ajuste (pelo critério dos mínimos quadrados) entre os pontos medidos, é improvável que a linha ajustada seja executada fora do valor medido mínimo ou máximo, exceto uma vez que vai para além da área medida (isto é, a extrapolação) (EPA, 2004; Naoum e Tsanis, 2004).

Emidio e Landim (2008) usaram a técnica da superfície de tendência com regressão polinomial de primeiro grau (superfície linear), para detectar anomalias na distribuição da chuva medida pelo radar meteorológico Doppler, banda S, localizado em Bauru-SP, no período de 21 de outubro de 2004 a 29 de abril de 2005. A técnica de análise de superfície de tendência também foi utilizada para estudar o comportamento espacial de alguns atributos do solo, ou seja, os locais onde ocorreram os menores e os maiores atributos. Por exemplo, a condutividade elétrica do solo e onde o solo pode ser mais favorável ao cultivo (Manfré et al., 2011). Urban et al. (2011) também utilizaram a mesma técnica no estudo de atributos do solo, apresentando-se potencial para verificação de impactos em áreas urbanas. Baseando-se nessa mesma técnica, o objetivo principal deste trabalho é utilizar o referido método como ferramenta para estimar médias mensais de precipitação para locais conhecidos (estações meteorológicas ou postos pluviométricos) e compará-las com valores medidos, com o intuito de verificar sua precisão dentro da região de estudos.

\section{MATERIAL E MÉTODOS}

Foram utilizados dados médios mensais de precipitação pluviométrica provenientes de estações meteorológicas e postos pluviométricos distribuídos espacialmente no estado da Paraíba, localizados em 102 municípios no período de 1962 a 2001 (Figura 1), cedidos pelo Instituto Nacional de Meteorologia (INMET). A priori foi feita uma análise descritiva dos dados e, em seguida, foram gerados os tipos de superfície de tendência com regressão polinomial de primeiro ao quinto grau. $\mathrm{Na}$ sequência, realizou-se a análise de variância (ANOVA) com objetivo de verificar qual tipo de regressão polinomial melhor se ajusta aos dados observados, também foram gerados mapas dos valores residuais entre os valores observados e os estimados pela regressão escolhida.

A análise de superfície de tendência é uma técnica simples e útil, visto que, tanto os mapas de tendência, quanto os residuais, podem ser interpretados do ponto de vista espacial. Essa técnica possibilita, também, efetuar interpolação para suprir a carência de dados observados. Neste estudo foram realizadas comparações entre os valores observados e estimados para os mesmos locais, utilizando-se o método dos polinômios não ortogonais. O modelo estatístico dos polinômios não ortogonais é definido pela seguinte equação:

$$
\begin{aligned}
z_{i}(X, Y)= & {\left[a_{0}+a_{1} x_{i}+a_{2} y_{i}+a_{3} x_{i}^{2}+a_{4} x_{i} y_{i}+a_{5} y_{i}^{2}+\ldots\right]+} \\
& +e_{i}\left(x_{i}, y_{i}\right)
\end{aligned}
$$




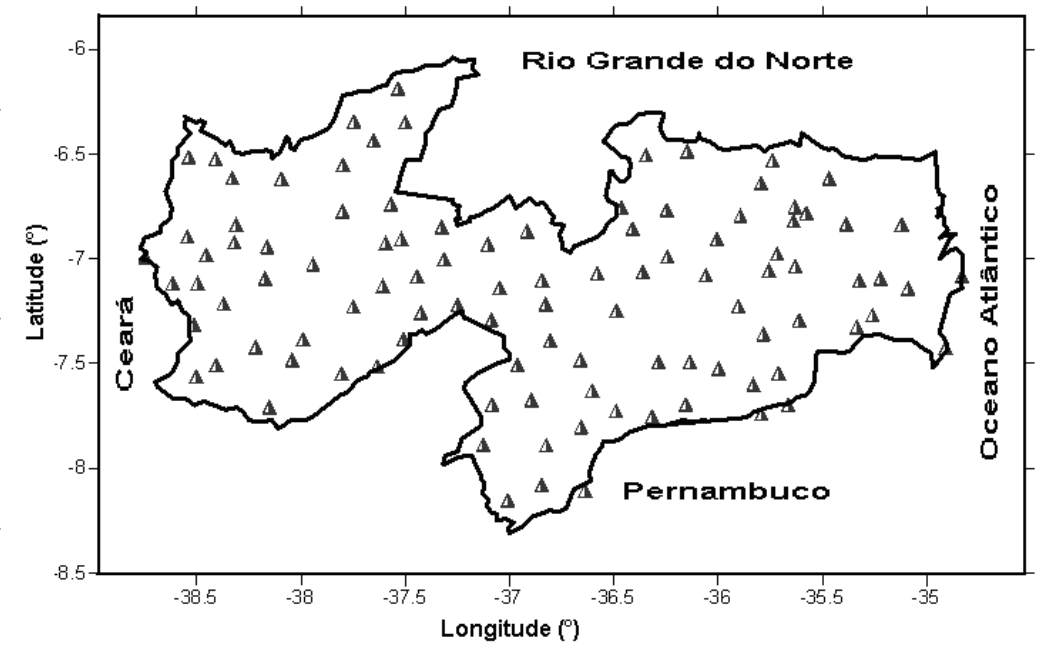

Figura 1 - Distribuição espacial dos 102 postos pluviométricos do Estado da Paraíba.

em que $z_{i}(X, Y)$ é a variável mapeada em função das coordenadas $x_{i}$ e $y_{i} ; e_{i}\left(x_{i}, y_{i}\right)$; representa os resíduos; $a_{0}, \ldots, a_{n}$ são os coeficientes que proporcionam o melhor ajuste aos dados amostrados. Os tipos de regressões polinomiais utilizados neste trabalho são apresentados nas Equações 2, 3, 4, 5 e 6.

$$
\begin{aligned}
z(X, Y)= & a_{0}+a_{1} x+a_{2} y \\
z(X, Y)= & a_{0}+a_{1} y+a_{2} y^{2}+a_{3} x+a_{4} x y+a_{5} x^{2} \\
z(X, Y)= & a_{0}+a_{1} y+a_{2} y^{2}+a_{3} y^{3}+a_{4} x+a_{5} x y+a_{6} x y^{2} \\
& +a_{7} x^{2}+a_{8} x^{2} y+a_{9} x^{3} \\
z(X, Y)= & a_{0}+a_{1} y+a_{2} y^{2}+a_{3} y^{3}+a_{4} y^{4}+a_{5} x+a_{6} x y+a_{7} x y^{2} \\
+ & a_{8} x y^{3}+a_{9} x^{2}+a_{10} x^{2} y+a_{11} x^{2} Y^{2}+a_{12} x^{3}+a_{13} x^{3} y+a_{14} x^{4} \\
z(X, Y)= & a_{0}+a_{1} y+a_{2} y^{2}+a_{3} y^{3}+a_{4} y^{4}+a_{5} y^{5}+a_{6} x+a_{7} x y \\
& +a_{8} x y^{2}+a_{9} x y^{3}+a_{19} x^{4} y+a_{20} x^{5}
\end{aligned}
$$

Conforme Landim (2003), a análise de variância (ANOVA) pode ser calculada por meio das seguintes equações:

$$
\begin{aligned}
& S Q T=\sum z_{i}^{2}-\left[\frac{\left(\sum z_{i}\right)^{2}}{n}\right] \\
& S Q P=\sum z^{*^{2}}{ }_{i}-\left[\frac{\left(\sum z_{i}^{*}\right)^{2}}{n}\right]
\end{aligned}
$$$$
S Q R=S Q T-S Q P
$$

em que $n$ é o número de observações, $S Q T$ é a variação total dos dados observados, $S Q P$ é a variação dos dados devido à superfície calculada e $S Q R$ é a variação devido aos resíduos.

Averificação doajuste das superfícies de tendênciaaos dados observados pode ser realizada testando-se as seguintes hipóteses:
$H_{0}$ : a variância dos dados estimados pela superfície é igual a dos dados originais, ou seja, não ocorre ajuste significativo da superfície aos dados.

$H_{1}$ :a variância dos dados estimados pela superfície é menor do que a dos dados originais, ou seja, ocorre ajuste significativo da superfície aos dados.

Uma estatística bastante utilizada em análise de superfície de tendência é o coeficiente de determinação, pois o mesmo é capaz de medir a porcentagem de ajuste da superfície. Segundo Landim (2003), o coeficiente de determinação pode ser definido pela porcentagem do ajuste da superfície obtida pela seguinte equação:

$$
R^{2}=\left(\frac{S Q P}{S Q T}\right) \times 100 \%
$$

A análise descritiva das médias mensais da precipitação foi realizada com o intuito de resumir as informações dos dados e descrevê-los em termos numéricos. Assim, depois de obtidas as medidas de tendência central e medidas de dispersão adotaram-se os critérios propostos por Oliveira (1999) na escolha do coeficiente de variação $\left(C_{v}\right)$, conforme definido pela Equação 11.

$$
C_{v}=\frac{S_{X}}{\bar{X}}
$$

em que $S_{X}$ e $\bar{X}$ representam, respectivamente, o desvio padrão e a média geral dos valores amostrais. O $C_{v}$ pode ser interpretado da seguinte forma: se o $C_{v}<10 \%$ indica baixa dispersão; entre $10 \%<C_{v}<20 \%$ indica média dispersão e $C_{v}>20 \%$ indica alta dispersão.

Outra medida de assimetria muito importante é o coeficiente de curtose ( $C_{k}$ ) que mede o grau de achatamento da amostra em relação à distribuição Normal (Borges, 2003). Os critérios de escolha do $C_{k}$ foram analisados de acordo 
Tabela 1 - Estatística descritiva das médias mensais da precipitação pluviométrica no período de 1962 a 2001 no estado da Paraíba.

\begin{tabular}{lcccccccc}
\hline Meses & Média & Mediana & Mínimo & Máximo & $\mathrm{S}$ & $\mathrm{C}_{\mathrm{S}}$ & $\mathrm{C}_{\mathrm{k}}$ & $\mathrm{C}_{\mathrm{v}} \%$ \\
\hline Janeiro & 66,24 & 58,26 & 16,37 & 159,96 & 36,18 & 0,64 & $-0,58$ & 54,62 \\
Fevereiro & 100,20 & 99,27 & 28,73 & 197,82 & 44,05 & 0,39 & $-0,91$ & 43,97 \\
Março & 160,65 & 159,09 & 67,30 & 396,22 & 57,73 & 0,67 & 1,28 & 35,94 \\
Abril & 153,70 & 155,05 & 76,92 & 321,31 & 48,29 & 0,64 & 0,57 & 31,42 \\
Maio & 83,51 & 75,11 & 29,78 & 287,84 & 45,55 & 2,10 & 6,56 & 54,55 \\
Junho & 63,40 & 36,33 & 10,80 & 322,79 & 60,95 & 2,31 & 5,86 & 96,14 \\
Julho & 57,78 & 29,34 & 3,23 & 303,27 & 62,12 & 1,93 & 3,56 & 107,52 \\
Agosto & 27,25 & 10,92 & 0,00 & 171,56 & 36,74 & 2,19 & 4,71 & 134,81 \\
Setembro & 16,94 & 6,80 & 0,00 & 103,19 & 22,04 & 2,00 & 3,43 & 130,09 \\
Outubro & 9,83 & 7,25 & 0,35 & 54,23 & 9,67 & 2,50 & 7,47 & 98,46 \\
Novembro & 13,38 & 11,18 & 1,26 & 54,89 & 9,92 & 1,61 & 3,49 & 74,11 \\
Dezembro & 30,27 & 28,60 & 7,65 & 76,18 & 13,75 & 0,75 & 0,19 & 45,42 \\
\hline
\end{tabular}

n: número de observações $(n=102)$; : desvio-padrão; $C_{\mathrm{v}}$ : coeficiente de variação; $\mathrm{C}_{\mathrm{S}}$ : coeficiente de assimetria; $\mathrm{C}_{\mathrm{k}}$ : coeficiente de curtose.

com Ribeiro Júnior (2004), conforme apresentado na seguinte equação:

$$
\begin{aligned}
& C_{k}=\frac{n \times(n+1)}{(n-1) \times(n-2) \times(n-3)} \\
& \times \sum_{i=1}^{n}\left(\frac{X_{i}-\bar{X}}{S_{X}}\right)^{4}-\frac{3 \times(n-1)^{2}}{(n-2) \times(n-3)}
\end{aligned}
$$

em que $n$ é o tamanho da amostra; $X_{i}$ são os valores observados; $\bar{X}$ é a média aritmética dos valores observados; $S_{X}$ é o desvio padrão da amostra. Se $C_{k}=0$ sugere que a distribuição é mesocúrtica, se $C_{k}<0$, a distribuição é leptocúrtica e se $C_{k}>$ 0 a distribuição é platicúrtica.

$\mathrm{O}$ coeficiente de assimetria $\left(\mathrm{C}_{\mathrm{s}}\right)$ é outra medida importante, pois, indica o grau de distorção da distribuição em relação a uma distribuição simétrica, isto é, numa distribuição simétrica os dados mais frequentes concentram-se mais ao centro em relação aos extremos. $\mathrm{O}_{\mathrm{s}}$ foi calculado de acordo com Equação 13.

$$
C_{s}=\frac{n}{(n-1) \times(n-2)} \times \sum_{i=1}^{n}\left(\frac{X_{i}-\bar{X}}{S_{X}}\right)^{3}
$$

em que $n$ é o tamanho da amostra; $X_{i}$ são os valores observados; $\bar{X}$ é a média aritmética dos valores observados; $S_{X}$ é o desvio padrão da amostra. Os critérios de escolha podem ser classificados da seguinte forma: para valores de $C_{s}=0$ a distribuição é dita simétrica; para valores de $C_{s}<0$ a distribuição é assimétrica negativa; para valores de $C_{s}>0$ a distribuição é assimétrica positiva.

\section{RESULTADOS E DISCUSSÃO}

Podem-se observar por meio da Tabela 1 os resultados da análise descritiva das médias mensais da precipitação. De acordo com os resultados, todos os meses apresentaram coeficiente de assimetria positivo $\left(C_{s}>0\right)$. Os coeficientes de variação $\left(C_{v}>20 \%\right)$ indicam que há alta dispersão entre os valores médios de precipitação, ou seja, há grande variabilidade da chuva no Estado da Paraíba, corroborando com os resultados

Tabela 2 - Coeficientes de determinação $\left(\mathrm{R}^{2}\right)$ referentes às superfícies calculadas para os diferentes meses do ano.

\begin{tabular}{lccccc}
\hline & $\begin{array}{c}\mathrm{R}^{2}(1) \\
(\%)\end{array}$ & $\begin{array}{c}\mathrm{R}^{2}(2) \\
(\%)\end{array}$ & $\begin{array}{c}\mathrm{R}^{2}(3) \\
(\%)\end{array}$ & $\begin{array}{c}\mathrm{R}^{2}(4) \\
(\%)\end{array}$ & $\begin{array}{c}\mathrm{R}^{2}(5) \\
(\%)\end{array}$ \\
\hline Janeiro & 55,73 & 80,73 & 85,25 & 87,47 & 87,66 \\
Fevereiro & 60,81 & 73,07 & 82,11 & 84,21 & 84,54 \\
Março & 48,68 & 55,98 & 71,68 & 74,62 & 74,74 \\
Abril & 23,95 & 37,77 & 64,16 & 67,04 & 67,16 \\
Maio & 17,69 & 50,97 & 73,79 & 75,78 & 75,79 \\
Junho & 47,06 & 74,22 & 80,00 & 81,07 & 81,08 \\
Julho & 57,16 & 79,00 & 81,48 & 82,73 & 82,80 \\
Agosto & 50,69 & 71,60 & 74,23 & 75,61 & 75,73 \\
Setembro & 46,80 & 76,05 & 78,80 & 80,50 & 80,58 \\
Outubro & 17,92 & 57,34 & 66,31 & 70,45 & 70,83 \\
Novembro & 2,64 & 47,31 & 56,58 & 62,08 & 62,42 \\
Dezembro & 18,88 & 57,74 & 62,24 & 65,56 & 66,17 \\
\hline
\end{tabular}



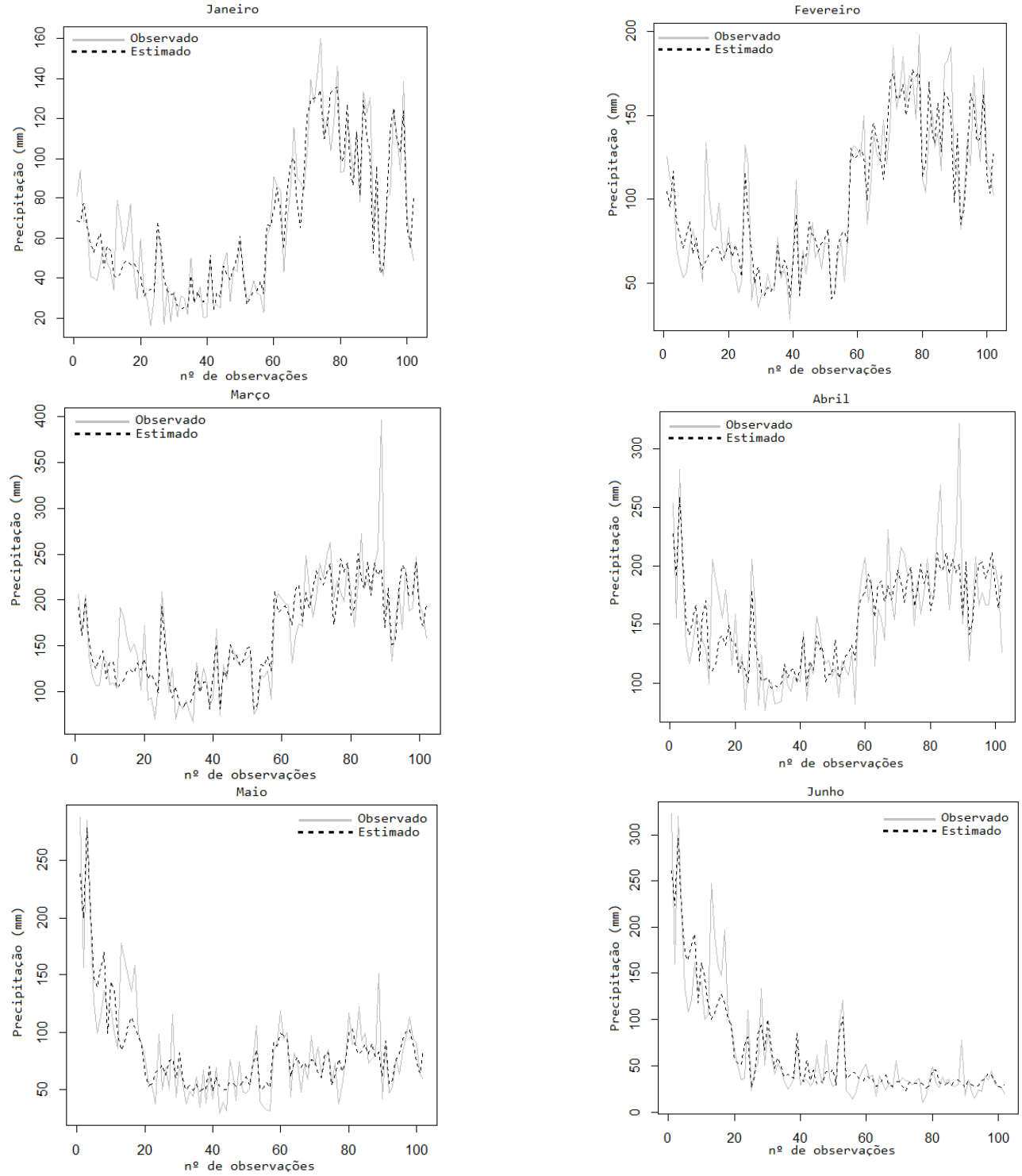

Figura 2 - Comparação entre os valores observados e os estimados pela superfície de $4^{\mathrm{a}}$ ordem (linha cheia corresponde aos valores observados e a linha pontilhada aos valores estimados). Os números apresentados nos eixos das ordenadas (x) correspondem aos números atribuídos a cada posto pluviométrico ou estação meteorológica.

encontrados por Silva et al. (2003), Menezes et al. (2010) e Macedo et al. (2010). Todos os valores médios são maiores do que as medianas, isso confirma a assimetria positiva dos dados. Com relação à curtose, apenas os valores médios mensais de janeiro e fevereiro apresentaram coeficientes de curtose $\left(C_{k}<\right.$ 0 ), indicando que nesses meses a distribuição dos dados é do tipo leptocúrtica, ou seja, significa que é mais fácil obter valores que se afastam da média, isso acontece devido nesses meses dar-se início a estação chuvosa da maior parte do estado, conforme descrito por Silva et al. (2009).

Os coeficientes de determinação das regressões polinomiais estão expostos na Tabela 2, onde de acordo com os resultados obtidos a superfície que apresentou a maior correlação com os dados observados foi a de maior ordem. Entretanto, ao aplicar a análise de variância (ANOVA), Tabelas 3 e 4, constata-se, pelo teste F, que as superfícies cúbica e quadrática se ajustaram bem aos valores médios mensais de precipitação. Sendo que, para os meses de janeiro, fevereiro, março, outubro e novembro a regressão polinomial que melhor se ajustou aos dados foi a de $4^{\circ}$ grau, para o restante dos meses o melhor ajuste foi a regressão polinomial de $3^{\circ}$ grau. Também foi feita a comparação entre os valores observados e os valores estimados pela regressão de $3^{\mathrm{a}}$ e $4^{\mathrm{a}}$ ordem apresentada nas Figuras 2 e 3. Pode-se observar que, em geral, os valores estimados se aproximaram consideravelmente dos valores observados, ou seja, apresentando valores estimados próximos 

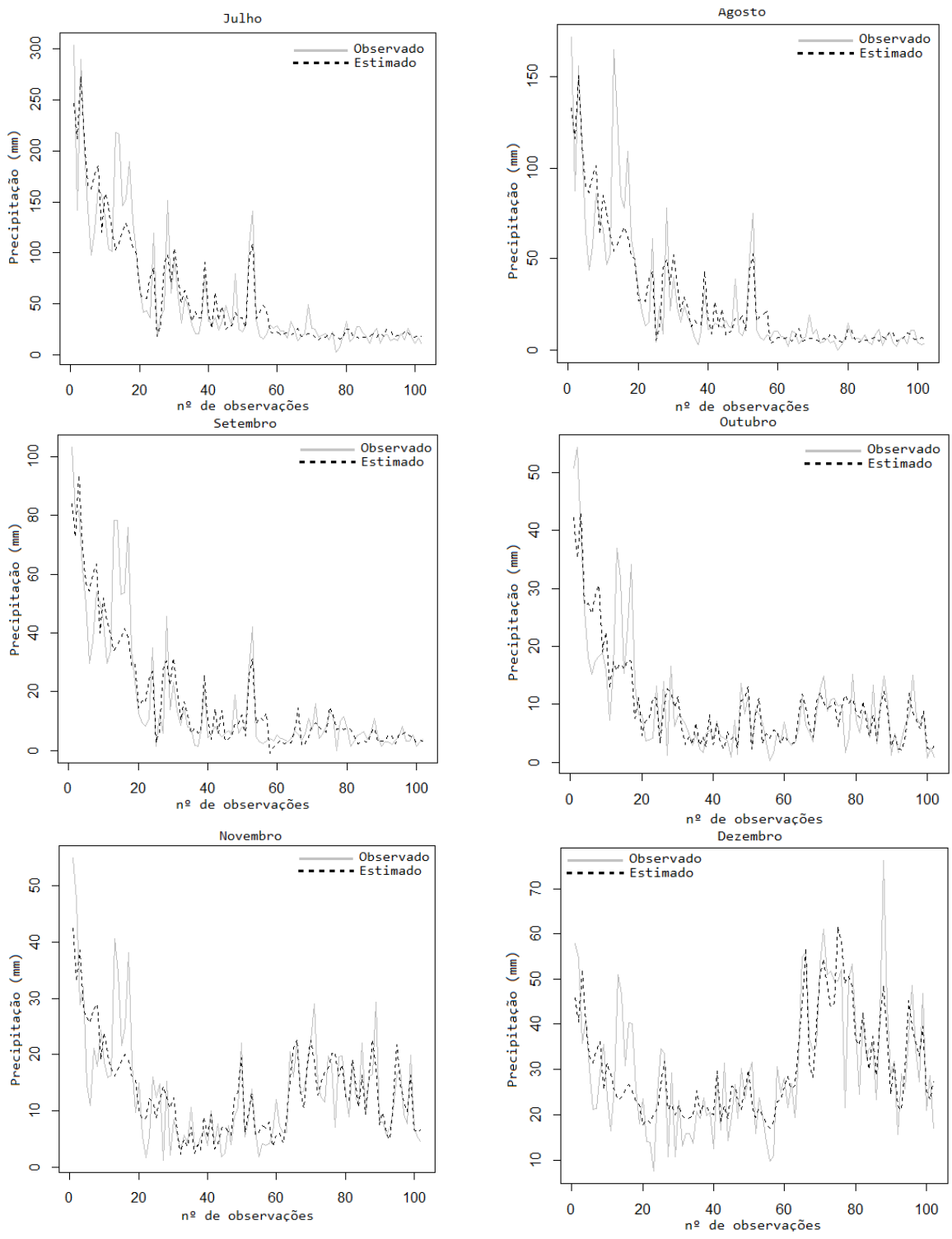

Figura 3 - Comparação entre os valores observados e os estimados pela superfície de $3^{\mathrm{a}} \mathrm{e} 4^{\mathrm{a}}$ ordem (a linha cheia corresponde aos valores observados e a linha pontilhada aos valores estimados). Os números apresentados nos eixos das ordenadas (x) correspondem aos números atribuídos a cada posto pluviométrico ou estação meteorológica.

dos observados, indicando-se que a superfície escolhida pode ser ainda mais precisa para se obter estimativas de precipitação mensal. Isso se deve porque nesses locais o regime de chuva é bastante similar durante alguns meses do ano, ocasionando-se numa maior uniformidade de chuva na região, conforme pode ser observado nos estudos de Silva et al. (2003), Silva et al. (2009), Menezes et al. (2010) e Macedo et al. (2010).

Os mapas dos resíduos padronizados, apresentados na Figura 4, indicam os locais onde ocorreram as maiores e menores diferenças entre os valores observados e estimados pela regressão polinomial de $3^{\mathrm{a}}$ e $4^{\mathrm{a}}$ ordem, pode-se observar que $95 \%$ das diferenças residuais encontram-se no intervalo de -2 a 2. Esses valores podem ser vistos em milímetros de precipitação mensal como apresentado na Figura 5. De acordo com os resultados, os meses de maio a novembro foram os que apresentaram as menores diferenças de precipitação entre os valores observados e estimados na maior parte da Paraíba (Figura 5), que vai do agreste ao Sertão (Figura 4). Vale salientar que, de acordo com Silva et al. (2009), durante o período supracitado, essa região apresenta baixo índice de precipitação, o que torna seu comportamento mais uniforme. Os modelos de regressão de $3^{\mathrm{a}}$ e $4^{\mathrm{a}}$ ordens se ajustaram bem a esse comportamento, conforme visto na Figura 4 e Tabela 2. Já nas regiões do Litoral e Brejo paraibano se concentram as maiores diferenças de precipitação devido à alta variabilidade da chuva (Figura 4), que está associada aos efeitos da brisa marítima e 

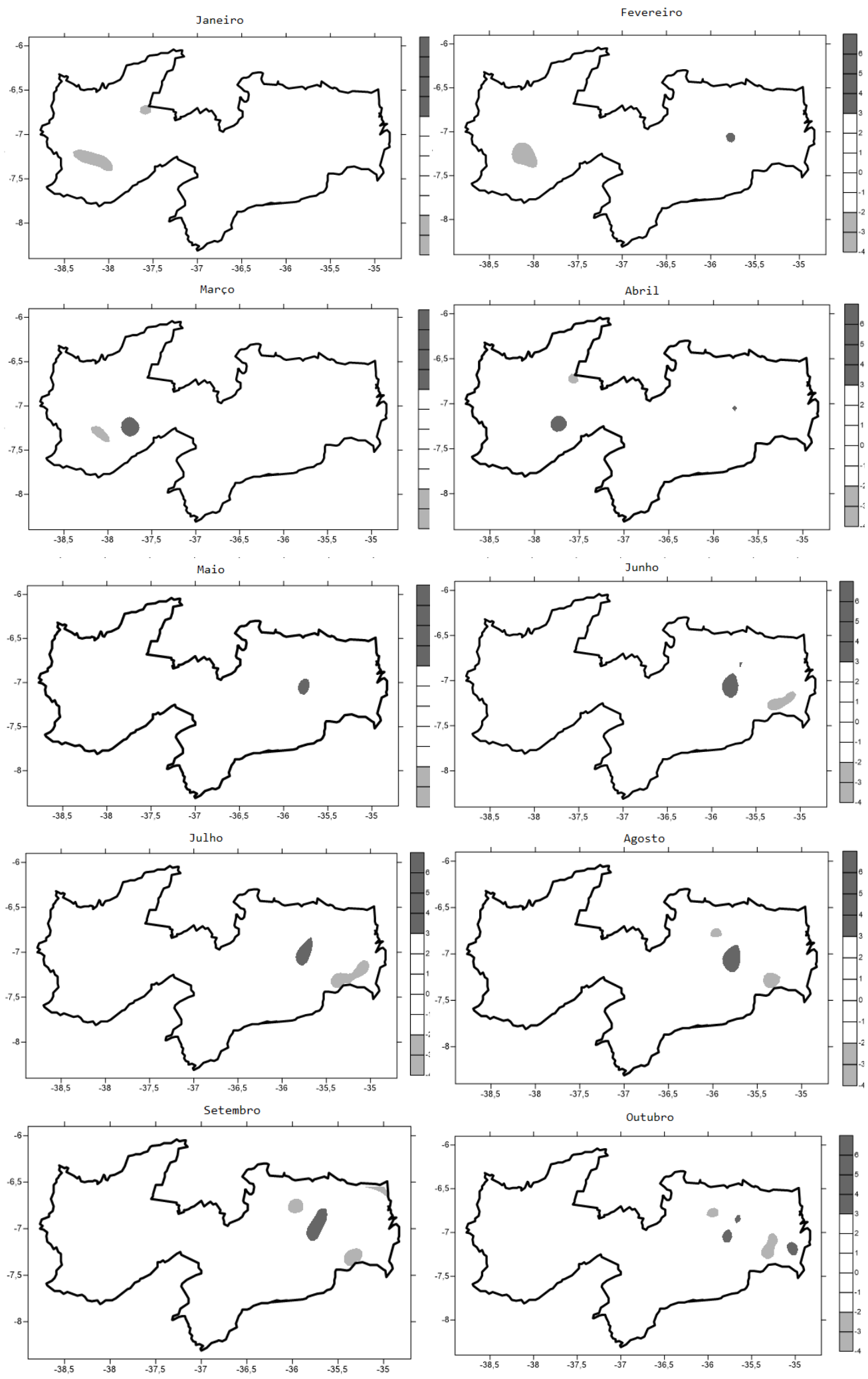

Figura 4 - Mapas dos resíduos, ou seja, das diferenças entre os valores estimados pela superfície de $3^{\mathrm{a}}$ e $4^{\mathrm{a}}$ ordem e os observados nos postos pluviométricos no estado da Paraíba. 

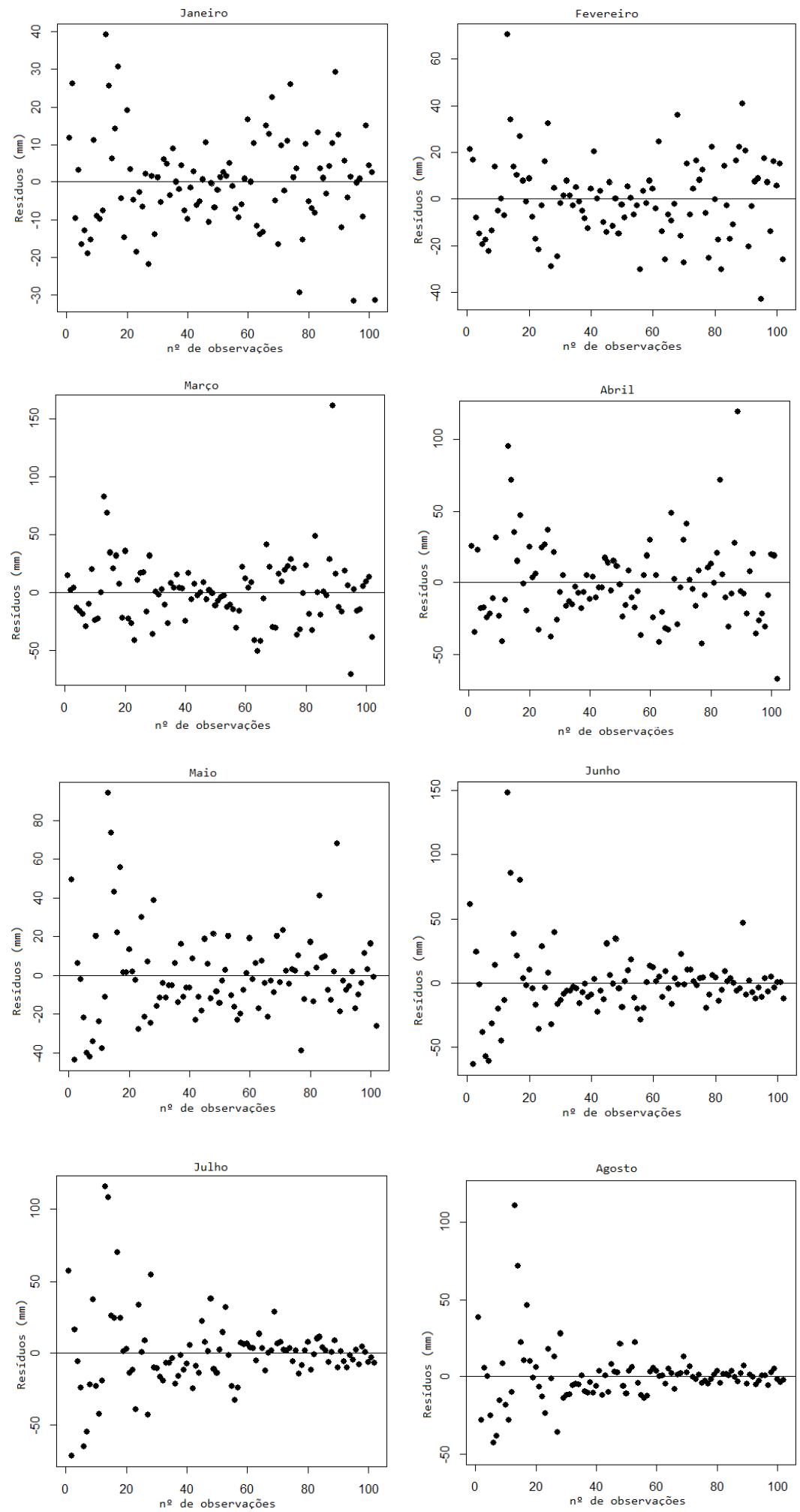

Figura 5 - Gráfico dos resíduos, ou seja, das diferenças entre os valores observados e estimados pelas superfícies de $3^{\mathrm{a}}$ e $4^{\mathrm{a}}$ ordem dos 102 postos pluviométricos no estado da Paraíba. Os números apresentados nos eixos das ordenadas (x) correspondem aos números atribuídos a cada posto pluviométrico ou estação meteorológica. (Continua). 

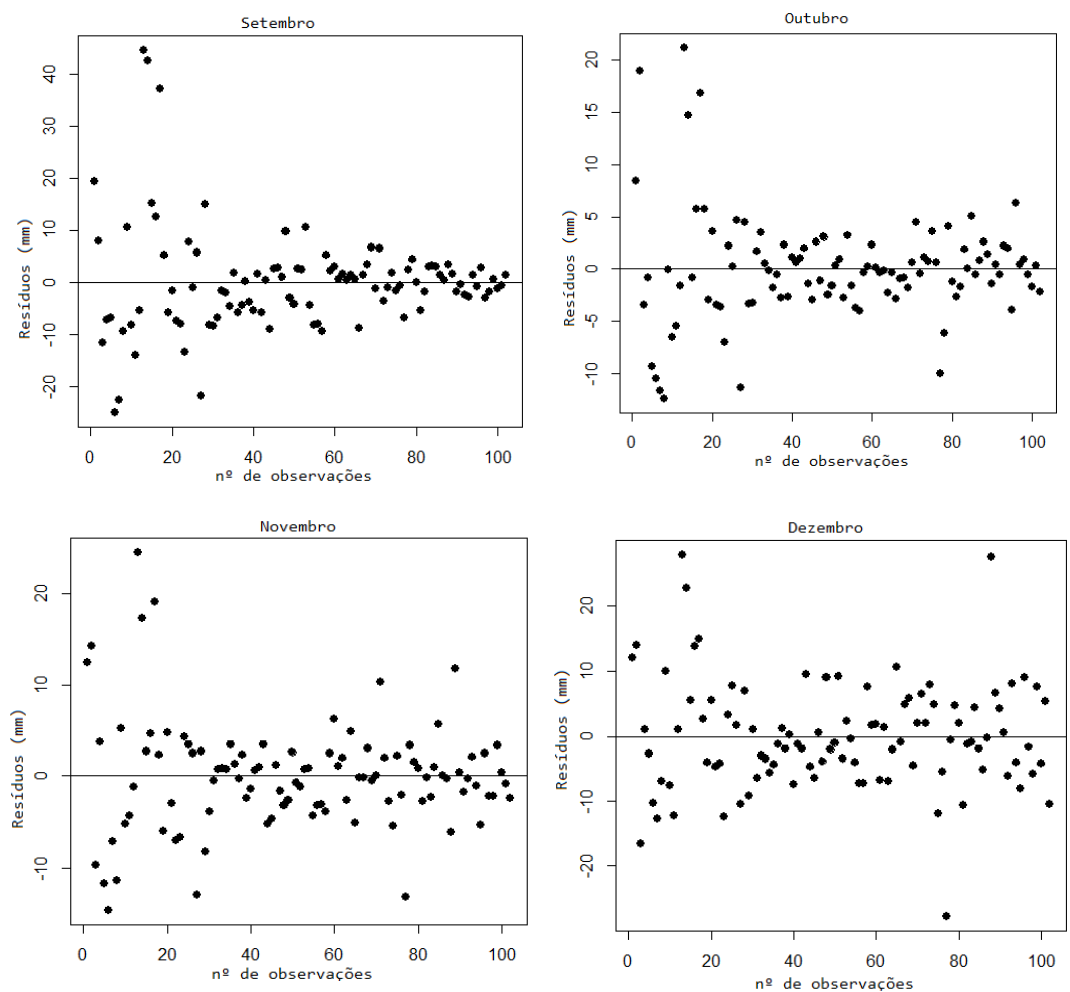

Figura 5 - Conclusão.

orográficos locais (Macedo et al., 2010). Isso faz com que os valores estimados pela superfície de tendência se distanciem mais dos valores observados.

Nos meses de dezembro a maio ocorre o processo inverso, ou seja, as maiores diferenças se concentram na região do Sertão devido o período chuvoso na região, causando maiores diferenças entre os valores observados e os estimados. Esses resultados estão de acordo com os encontrados por Silva et al. (2003), Menezes et al. (2010) e Macedo et al. (2010). Esse período chuvoso apresenta alta variabilidade espacial devido à atuação de diferentes sistemas meteorológicos, tais como, ZCIT e VCAN, conforme descrito por Cavalcanti et al. (2009) e Macedo et al. (2010).

Dando sequência as análises, pode-se observar por meio das Tabelas 3 e 4 os resultados da análise de variância da regressão polinomial de $3^{\circ}$ e $4^{\circ}$ graus ajustada aos dados observados dos diferentes meses do ano, para os níveis de significância adotados $(\alpha=1 \%$ e $\alpha=5 \%)$. Com base nos valores críticos da estatística $\mathrm{F}$ (coeficiente de Fisher) para os níveis de significância de $1 \%$ e 5\%, com 9 e 92 graus de liberdade têm-se 2,61 e 1,98, e com 14 e 87 graus de liberdade tem-se 2,29 e 1,80 respectivamente. Como os valores calculados de $\mathrm{F}$ foram maiores do que os valores críticos; rejeita-se a hipótese nula $\left(\mathrm{H}_{0}\right)$, corroborando com a hipótese alternativa $\left(\mathrm{H}_{1}\right)$, isto é, a variância dos dados estimados pela regressão calculada é menor do que a dos dados originais, ou seja, o ajuste da regressão aos dados é considerado significativo. Isso indica que o modelo pode ser utilizado para fazer estimativas de precipitação média mensal para locais desconhecidos dentro da região estudada, ou para locais circunvizinhos das estações, onde os resultados seriam mais precisos.

\section{CONCLUSÕES}

De acordo com a análise descritiva dos dados pode se observar que existe grande variabilidade espacial da precipitação mensal em todo o Estado da Paraíba.

As regressões polinomiais de $3^{\mathrm{a}}$ e $4^{\mathrm{a}}$ ordem foram as que melhor se ajustaram aos dados de precipitação média mensal observada no Estado da Paraíba.

A comparação entre os valores medidos e estimados mostram que os meses de maio a novembro a região do Litoral apresentaram as maiores diferenças de precipitação em relação ao restante do Estado paraibano (do agreste ao Sertão) e, que os meses de dezembro a abril as maiores diferenças, concentram-se na região do Sertão. Isso evidencia que o modelo reduz sua precisão, quando aplicado aos referidos meses nessas localidades.

Portanto, o método da regressão polinomial (superfície de tendência) revelou-se como uma importante ferramenta 
Tabela 3 - Análise de variância para verificação da superfície de tendência do modelo de regressão polinomial ajustada às médias mensais de precipitação pluviométrica do estado da Paraíba. GLR = graus de liberdade dos resíduos, $\mathrm{SQR}$ = soma de quadrado do resíduos, Df é a diferença do incremento do grau de liberdade residual, $\mathrm{SQ}=$ soma de quadrados, $\mathrm{F}=$ teste de Fisher.

\begin{tabular}{|c|c|c|c|c|c|c|}
\hline Fonte de variação: janeiro & GLR & SQR & Df & SQ & $\mathrm{F}$ & $\operatorname{Pr}(>\mathrm{F})$ \\
\hline Regressão de $1^{\mathrm{a}}$ ordem & 99 & 58517 & & & & \\
\hline Regressão de $2^{\mathrm{a}}$ ordem & 96 & 25469 & 3 & 33048 & 57,402 & $<0,001 * *$ \\
\hline Regressão de $3^{\mathrm{a}}$ ordem & 92 & 19495 & 4 & 5974 & 7,782 & $<0,001 * *$ \\
\hline Regressão de $4^{\mathrm{a}}$ ordem & 87 & 16567 & 5 & 2928 & 3,051 & $0,014^{*}$ \\
\hline Regressão de $5^{\mathrm{a}}$ ordem & 85 & 16313 & 2 & 254 & 0,661 & 0,519 \\
\hline Fonte de variação: fevereiro & GLR & SQR & Df & SQ & $\mathrm{F}$ & $\operatorname{Pr}(>\mathrm{F})$ \\
\hline Regressão de $1^{\mathrm{a}}$ ordem & 99 & 76817 & & & & \\
\hline Regressão de $2^{\mathrm{a}}$ ordem & 96 & 52794 & 3 & 24023 & 22,458 & $<0,001 * *$ \\
\hline Regressão de $3^{\mathrm{a}}$ ordem & 92 & 35065 & 4 & 17729 & 12,429 & $<0,001 * *$ \\
\hline Regressão de $4^{\mathrm{a}}$ ordem & 87 & 30948 & 5 & 4117 & 2,309 & $0,050^{*}$ \\
\hline Regressão de $5^{\mathrm{a}}$ ordem & 85 & 30311 & 2 & 636 & 0,892 & 0,413 \\
\hline Fonte de Variação: março & GLR & $\mathrm{SQR}$ & Df & SQ & $\mathrm{F}$ & $\operatorname{Pr}(>\mathrm{F})$ \\
\hline Regressão de $1^{\mathrm{a}}$ ordem & 99 & 172772 & & & & \\
\hline Regressão de $2^{\mathrm{a}}$ ordem & 96 & 148196 & 3 & 24576 & 8,187 & $<0,001 * *$ \\
\hline Regressão de $3^{\mathrm{a}}$ ordem & 92 & 95348 & 4 & 52848 & 13,209 & $<0,001 * *$ \\
\hline Regressão de $4^{\mathrm{a}}$ ordem & 87 & 85435 & 5 & 9913 & 1,981 & $0,050 *$ \\
\hline Regressão de $5^{\mathrm{a}}$ ordem & 85 & 85052 & 2 & 383 & 0,192 & 0,825 \\
\hline Fonte de Variação: abril & GLR & SQR & Df & SQ & $\mathrm{F}$ & $\operatorname{Pr}(>\mathrm{F})$ \\
\hline Regressão de $1^{\mathrm{a}}$ ordem & 99 & 179134 & & & & \\
\hline Regressão de $2^{\mathrm{a}}$ ordem & 96 & 146582 & 3 & 32552 & 11,924 & $<0,001 * *$ \\
\hline Regressão de $3^{\mathrm{a}}$ ordem & 92 & 84427 & 4 & 62155 & 17,075 & $<0,001 * *$ \\
\hline Regressão de $4^{\mathrm{a}}$ ordem & 87 & 77640 & 5 & 6788 & 1,492 & 0,201 \\
\hline Regressão de $5^{\mathrm{a}}$ ordem & 85 & 77349 & 2 & 291 & 0,160 & 0,852 \\
\hline Fonte de Variação: maio & GLR & SQR & Df & SQ & $\mathrm{F}$ & $\operatorname{Pr}(>\mathrm{F})$ \\
\hline Regressão de $1^{\mathrm{a}}$ ordem & 99 & 172509 & & & & \\
\hline Regressão de $2^{\mathrm{a}}$ ordem & 96 & 102766 & 3 & 69743 & 38,934 & $<0,001 * *$ \\
\hline Regressão de $3^{\mathrm{a}}$ ordem & 92 & 54938 & 4 & 47827 & 20,025 & $<0,001 * *$ \\
\hline Regressão de $4^{\mathrm{a}}$ ordem & 87 & 50769 & 5 & 4170 & 1,396 & 0,233 \\
\hline Regressão de $5^{\mathrm{a}}$ ordem & 85 & 50753 & 2 & 16 & 0,013 & 0,987 \\
\hline Fonte de Variação: junho & GLR & SQR & Df & SQ & $\mathrm{F}$ & $\operatorname{Pr}(>\mathrm{F})$ \\
\hline Regressão de $1^{\mathrm{a}}$ ordem & 99 & 198655 & & & & \\
\hline Regressão de $2^{\mathrm{a}}$ ordem & 96 & 96758 & 3 & 101897 & 40,659 & $<0,001 * *$ \\
\hline Regressão de $3^{\mathrm{a}}$ ordem & 92 & 75060 & 4 & 21698 & 6,493 & $<0,001 * *$ \\
\hline Regressão de $4^{\mathrm{a}}$ ordem & 87 & 71045 & 5 & 4015 & 0,961 & 0,446 \\
\hline Regressão de $5^{\mathrm{a}}$ ordem & 85 & 71007 & 2 & 38 & 0,023 & 0,977 \\
\hline
\end{tabular}


Tabela 4 - Análise de variância para verificação da superfície de tendência do modelo de regressão polinomial ajustada às médias mensais de precipitação pluviométrica do estado da Paraíba. GLR = graus de liberdade dos resíduos, SQR = soma de quadrado do resíduos, Df é a diferença do incremento do grau de liberdade residual, $\mathrm{SQ}=$ soma de quadrados, $\mathrm{F}=$ teste de Fisher.

\begin{tabular}{|c|c|c|c|c|c|c|}
\hline Fonte de variação: julho & GLR & SQR & Df & SQ & $\mathrm{F}$ & $\operatorname{Pr}(>\mathrm{F})$ \\
\hline Regressão de $1^{\mathrm{a}}$ ordem & 99 & 166995 & & & & \\
\hline Regressão de $2^{\mathrm{a}}$ ordem & 96 & 81849 & 3 & 85146 & 35,989 & $<0,001 * *$ \\
\hline Regressão de $3^{\mathrm{a}}$ ordem & 92 & 72178 & 4 & 9671 & 3,066 & 0,021 \\
\hline Regressão de $4^{\mathrm{a}}$ ordem & 87 & 67335 & 5 & 4843 & 1,228 & 0,303 \\
\hline Regressão de $5^{\mathrm{a}}$ ordem & 85 & 67032 & 2 & 303 & 0,192 & 0,825 \\
\hline Fonte de variação: agosto & GLR & SQR & Df & SQ & $\mathrm{F}$ & $\operatorname{Pr}(>F)$ \\
\hline Regressão de $1^{\mathrm{a}}$ ordem & 99 & 67220 & & & & \\
\hline Regressão de $2^{\mathrm{a}}$ ordem & 96 & 38707 & 3 & 28513 & 24,421 & $<0,001 * *$ \\
\hline Regressão de $3^{\mathrm{a}}$ ordem & 92 & 35127 & 4 & 3579 & 2,299 & 0,065 \\
\hline Regressão de $4^{\mathrm{a}}$ ordem & 87 & 33242 & 5 & 1885 & 0,968 & 0,441 \\
\hline Regressão de $5^{\mathrm{a}}$ ordem & 85 & 33081 & 2 & 161 & 0,207 & 0,812 \\
\hline Fonte de variação: setembro & GLR & SQR & Df & SQ & $\mathrm{F}$ & $\operatorname{Pr}(>F)$ \\
\hline Regressão de $1^{\mathrm{a}}$ ordem & 99 & 26106 & & & & \\
\hline Regressão de $2^{\mathrm{a}}$ ordem & 96 & 11753 & 3 & 14352 & 42,662 & $<0,001 * *$ \\
\hline Regressão de $3^{\mathrm{a}}$ ordem & 92 & 10401 & 4 & 1352 & 3,014 & 0,022 \\
\hline Regressão de $4^{\mathrm{a}}$ ordem & 87 & 9571 & 5 & 830 & 1,481 & 0,204 \\
\hline Regressão de $5^{\mathrm{a}}$ ordem & 85 & 9531 & 2 & 39 & 0,174 & 0,840 \\
\hline Fonte de variação: outubro & GLR & SQR & Df & SQ & $\mathrm{F}$ & $\operatorname{Pr}(>\mathrm{F})$ \\
\hline Regressão de $1^{\mathrm{a}}$ ordem & 99 & 7758 & & & & \\
\hline Regressão de $2^{\mathrm{a}}$ ordem & 96 & 4032 & 3 & 3726 & 38,285 & $<0,001 * *$ \\
\hline Regressão de $3^{\mathrm{a}}$ ordem & 92 & 3184 & 4 & 848 & 6,534 & $<0,001 * *$ \\
\hline Regressão de $4^{\mathrm{a}}$ ordem & 87 & 2793 & 5 & 390 & 2,409 & $0,042 *$ \\
\hline Regressão de $5^{\mathrm{a}}$ ordem & 85 & 2757 & 2 & 35 & 0,552 & 0,577 \\
\hline $\begin{array}{l}\text { Fonte de variação: } \\
\text { novembro }\end{array}$ & GLR & SQR & Df & SQ & $\mathrm{F}$ & $\operatorname{Pr}(>F)$ \\
\hline Regressão de $1^{\mathrm{a}}$ ordem & 99 & 9667 & & & & \\
\hline Regressão de $2^{\mathrm{a}}$ ordem & 96 & 5231 & 3 & 4435 & 33,680 & $<0,001 * *$ \\
\hline Regressão de $3^{\mathrm{a}}$ ordem & 92 & 4312 & 4 & 919 & 5,237 & $<0,001 * *$ \\
\hline Regressão de $4^{\mathrm{a}}$ ordem & 87 & 3766 & 5 & 546 & 2,487 & $0,037^{*}$ \\
\hline Regressão de $5^{\mathrm{a}}$ ordem & 85 & 3731 & 2 & 34 & 0,392 & 0,676 \\
\hline $\begin{array}{l}\text { Fonte de variação: } \\
\text { dezembro }\end{array}$ & GLR & SQR & Df & SQ & $\mathrm{F}$ & $\operatorname{Pr}(>F)$ \\
\hline Regressão de $1^{\mathrm{a}}$ ordem & 99 & 15490 & & & & \\
\hline Regressão de $2^{\mathrm{a}}$ ordem & 96 & 8070 & 3 & 7419 & 32,541 & $<0,001 * *$ \\
\hline Regressão de $3^{\mathrm{a}}$ ordem & 92 & 7211 & 4 & 859 & 2,825 & $0,029 *$ \\
\hline Regressão de $4^{\mathrm{a}}$ ordem & 87 & 6577 & 5 & 634 & 1,668 & 0,151 \\
\hline Regressão de $5^{\mathrm{a}}$ ordem & 85 & 6460 & 2 & 117 & 0,771 & 0,465 \\
\hline
\end{tabular}


para estimar dados de precipitação pluvial mensal na área de estudo.

\section{AGRADECIMENTOS}

Ao INMET pela concessão dos dados de precipitação pluvial, a CAPES pela concessão da bolsa de estudo ao primeiro autor e ao $\mathrm{CNPq}$ pelas bolsas de Produtividade em Pesquisa dos segundo e terceiro autores. Adicionalmente, os autores agradecem aos revisores pelas suas valiosas correções e sugestões.

\section{REFERÊNCIAS BIBLIOGRÁFICAS}

BORGES, B. L. M. Simplificando a Estatística. 2. Ed. Campina Grande: EDUEP, 2003, 208p. ISBN 85-87108-16-6.

CAVALCANTI, I. F. A.; FERREIRA, N. J.; DIAS, M. A. F.; JUSTI, M. G. A. (Org.). Tempo e Clima no Brasil. Ed. Oficina de Textos, $1^{\text {a }}$ Ed., 464p., 2009.

EMIDIO. Z. P. O; LANDIM. P. M. B. Análise de superfície de tendência aplicada à chuva, medida por radar meteorológico, nas regiões de assis e piracicaba, SP. Geociências, v.27, n.4, p.439 - 449, 2008.

EPA (U.S. ENVIRONMENTAL PROTECTION AGENCY). Developing Spatially Interpolated Surfaces and Estimating Uncertainty. Report. EPA-454/R-04-004, 169 p. 2004.

LANDIM, P. M. B. Análise Estatística de Dados Geológicos. 2. ed. São Paulo: Unesp, 2003, 253p. ISBN 85-7139-504-7.

LANDIM. P. M. B; CORSI. A. C. Cálculo de superfície de tendência, por regressão polinomial, pelo SURFER 6 . DGA, IGCE, UNESP/Rio Claro, lab, Geomatemática, Texto Didático 05. Rio Claro: UNESP, 11p. 2001. Disponível em <http://www.solos.ufmt.br/docs/geoestatistica/ calctendsurfer.pdf $>$ Acesso em 03 de Outubro. 2011.

MACEDO, M. J. H.; GUEDES, R. V. S.; SOUSA, F. A. S.; DANTAS, F. R. C. Análise do índice padronizado de precipitação para o estado da Paraíba, Brasil. Revista Ambiente \& Água, v. 5, n. 1, p. 204-214, 2010.
MANFRÉ. L. A et al. Análise de superfície de tendência de variáveis de solo como subsídio ao planejamento ambiental de uma bacia hidrográfica rural, XV Simpósio Brasileiro de Sensoriamento Remoto, Curitiba - PR, Brasil, 2011.

MENEZES, H. E. A.; BRITO, J. I. B.; LIMA, R. A. F. A. Veranico e a produção agrícola no Estado da Paraíba, Brasil. Revista Brasileira de Engenharia Agrícola e Ambiental, v. 14, p. 181-186, 2010.

NAOUM, S., TSANIS, I. K. Ranking spatial interpolation techniques using a GIS-based DSS. Global NEST Journal, v. 6, p. 1-20, 2004.

OLIVEIRA, F.E.M. Estatística e Probabilidade. 2. ed. São Paulo: Atlas, 1999, 221p. ISBN 85-224-2103-X.

PEDROZA, I, C, B. Estimativa da Climatologia Diária da Precipitação e Investigação de Possíveis Influências das Fases da Lua nas Chuvas no Estado da Paraíba: Campina Grande, 2009. 86p. Dissertação (Mestrado em Meteorologia). Universidade Federal de Campina Grande.

RIBEIRO JÚNIOR, J. I. Análises Estatísticas no EXCEL. Viçosa-MG, 2004. 251p. ISBN 85-7269-175-8.

RODRIGUEZ, J, L. Atlas Escolar da Paraíba, 3. ed.João Pessoa: Grafset, 2002. 112p. ISBN 85-85893-05-2.

SILVA, L. L.; COSTA, R. F., CAMPOS, J. H. B. C.; DANTAS,

R. T. Influência das precipitações na produtividade agrícola no Estado da Paraíba. Revista Brasileira de Engenharia Agrícola e Ambiental, v.13, n.4, p.454-461, 2009.

SILVA, V. P. R.; CAVALCANTI, E. P.; NASCIMENTO, M. G.; CAMPOS, J. H. B. C. Análises da precipitação pluvial no Estado da Paraíba com base na teoria da entropia. Revista Brasileira de Engenharia Agrícola e Ambiental, v.7, n.2, p.269-274, 2003.

URBAN. R. C et al. Análise de superfície de tendência de variáveis de solo prioritariamente urbano, XV Simpósio Brasileiro de Sensoriamento Remoto, Curitiba - PR, Brasil, 2011. 